\title{
Transcranial laser therapy for acute ischemic stroke: a pooled analysis of NEST-1 and NEST-2
}

\author{
Branko N Huisa ${ }^{1}$, Andrew B Stemer ${ }^{2}$, Michael G Walker ${ }^{3}$, Karen Rapp ${ }^{2}$, Brett C. Meyer ${ }^{2}$, and \\ Justin A Zivin ${ }^{2}$ for the NEST-1 and -2 investigators \\ ${ }^{1}$ Department of Neurology, University of New Mexico, NM \\ ${ }^{2}$ Department of Neurosciences, University of California San Diego School of Medicine, San \\ Diego, CA \\ ${ }^{3}$ Department of Medicine, Stanford University, Palo Alto, CA
}

\begin{abstract}
Background-NeuroThera Effectiveness and Safety Trials (NEST) 1 and 2 have demonstrated safety of transcranial laser therapy for human treatment in acute ischemic stroke. The NEST-1 study suggested efficacy of transcranial laser therapy but the following NEST-2, despite strong signals, missed reaching significance on its primary efficacy endpoint. In order to assess efficacy in a larger cohort, a pooled analysis was therefore performed.
\end{abstract}

Methods-The two studies were first compared for heterogeneity, and then a pooled analysis was performed to assess overall safety and efficacy, and examined particular subgroups. The primary endpoint for the pooled analysis was dichotomized modified Rankin scale (mRS 0-2) at 90 days.

Results-Efficacy analysis for the Intention-To-Treat population was based on a total of 778 patients. Baseline characteristics and prognostic factors were balanced between the two groups. The transcranial laser therapy group $(n=410)$ success rate measured by the dichotomized 90 day mRS was significantly higher compared with the Sham group $(\mathrm{n}=368)(\mathrm{p}=0.003$, OR $1.67,95 \%$ CI 1.19-2.35). The distribution of scores on the 90-day mRS was significantly different in transcranial laser therapy compared with Sham ( $\mathrm{p}=0.0005$ Cochran-Mantel-Haenszel). Subgroup analysis identified moderate strokes as a predictor of better treatment response.

Conclusions-This pooled analysis support the likelihood that transcranial laser therapy is effective for the treatment of acute ischemic stroke when initiated within $24 \mathrm{~h}$ of stroke onset. If ultimately confirmed, transcranial laser therapy will change management for ischemic stroke and improve outcomes of far more patients with acute ischemic stroke.

\section{Keywords}

Clinical trial; stroke outcomes; neuroprotection; acute stroke; laser therapy

Correspondence: Branko N Huisa, M.D. Department of Neurology, MSC10 5620, 1UNM, Albuquerque, NM, 87131. bhuisa@unm.edu.

Conflict of interest

Disclosures

$\mathrm{BH}, \mathrm{AS}, \mathrm{KR}$ and BM have nothing to disclose.

MW is a consultant for and stockholder in Photothera and JZ is a consultant for Photothera. 


\section{Introduction}

The only proven treatment for acute ischemic stroke is intravenous thrombolysis with tissue plasminogen activator (t-PA) when given within $4.5 \mathrm{~h}$ of stroke onset. ${ }^{1,2}$ With nearly 800 000 new strokes a year in the US, and fewer than $5 \%$ treated with thrombolytics it's evident that determining alternative acute stroke treatments is imperative. The brief window of time for administration, the ever-present risk of bleeding, and the need for significant education and training in the appropriate use of thrombolytics are some of the most important barriers to increasing t-PA use. ${ }^{3,4}$ Newer and safer treatments, potentially with longer treatment windows would translate to more patients receiving treatment. Unfortunately the quest for neuroprotective treatments in acute ischemic stroke, to date, has so far been unsuccessful. ${ }^{5}$

Transcranial laser therapy (TLT) is a noninvasive technology that uses near-infrared laser energy transmitted through the scalp and skull to modulate biological changes within brain cells. ${ }^{6}$ The mechanism of TLT appears to be multifactorial. It is likely a combination of improved energy production ${ }^{7}$, neuroprotection ${ }^{8}$, neurogenesis ${ }^{9}$ and neuroplasticity. ${ }^{10}$ Preclinical studies with TLT applied to different stroke models have produced encouraging results. ${ }^{9,11-13}$ Each model exhibited behavior that was significantly improved as early as 24 $\mathrm{h}$ after treatment and was sustained for the duration of the study. Equally important was the observation that TLT appeared effective even when applied as late as $24 \mathrm{~h}$ from stroke onset, while maintaining a benign side effect profile. ${ }^{14}$ The first human trial of TLT, NeuroThera Effectiveness and Safety Trial-1 (NEST-1), was a prospective, double blind, 2:1 randomized, sham-controlled study that enrolled 120 patients (79 patients receiving TLT therapy) within $24 \mathrm{~h}$ of stroke symptom onset. Its primary efficacy outcome, binary NIHSS (bNIH), defined as a complete recovery at day 90 (NIHSS 0 to 1) or a decrease in NIHSS score of at least 9 points (day 90 versus baseline), was statistically significant (along with the secondary outcome of binary $\mathrm{mRS}(0-2=$ success $))$ without any serious adverse events (SAEs) related to either the device or procedure. ${ }^{14}$ The subsequent NEST 2 clinical trial was a larger 1:1 randomized, double blind, sham-controlled study with 660 subjects (331 TLT, 329 sham). ${ }^{15}$ NEST-2 expanded the enrollment age range to $40-90$ years (40-85 for NEST-1), and used the binary mRS as the primary efficacy endpoint. Unlike NEST-1, NEST-2 did not reach statistical significance for efficacy $(\mathrm{p}=0.09)$ but did confirm the safety of the therapy. ${ }^{15}$ NEST-1 and 2, both yielded promising, yet non-equivalent results with respect to their primary efficacy outcomes. The variations in efficacy may have been due to inclusion/exclusion differences, limitations in sample size, differences in baseline characteristics or any combination of these factors. We sought to determine whether a pooled analysis would provide further evidence of efficacy in a larger cohort of patients, and elucidate elements, which may identify patient groups most amenable to TLT benefit.

\section{Patients and methods}

\section{Study design}

NEST-1 and 2 enrolled and randomized a total of 780 patients at 61 centers in five countries. NEST1 recruited subjects from Israel, Peru and Sweden, and NEST 2 subjects were from Peru, Sweden, Germany and the United States. Both studies were conducted in accordance with FDA/ICH Good Clinical Practice guidelines; local governing authorities, and ethics committees/ institutional review boards approved protocols. The design and conduct of both NEST studies were done in conjunction with a steering committee (SC) comprising stroke experts from Europe and North America. The SC had complete access to all data and was responsible for drafting the manuscripts. The sponsor, Photothera, was responsible for operational aspects of the trial, including collecting and storing the data and performing the analysis according to the approved statistical analysis plan. 


\section{Patient selection}

Inclusion criteria for both clinical trials were similar. In both trials, patients were required to have a clinical diagnosis of acute ischemic stroke with a clearly defined onset time, and both trials required initiation of treatment within $24 \mathrm{~h}$ of symptom onset. Eligible patients were between 40-85 years of age (NEST-1) or 40-90 years of age (NEST-2), and had presenting NIHSS scores of 7-22 (inclusive). Baseline (pre-stroke) mRS was not obtained for NEST-1, but was required to be $\leq 2$ for NEST-2.

Exclusion criteria for both clinical trials were also similar. Patients were excluded if there was evidence of or suspicion of intracranial hemorrhage or if they were candidates for thrombolysis. Patients were not enrolled if they had a seizure at stroke onset, blood glucose $<40 \mathrm{mg} / \mathrm{dL}$ or $>400 \mathrm{mg} / \mathrm{dL}$, sustained blood pressure $>185 / 110 \mathrm{mmHg}$, known history of bleeding disorder, oral anticoagulation with elevated prothrombin time, a significant skin condition on the scalp, severe mental deficit, neurological disorder which could interfere with further assessments, or if the patient was otherwise deemed to be medically unsuitable. Randomization was distinct for each trial. Subjects were randomized in a 2:1 fashion in NEST-1 and 1:1 in NEST-2. Since the inclusion/exclusion criteria and therapeutic procedures were similar for both of the NEST studies (with a notable exception of age limit in NEST-2), pooling the individual patient data to assess the efficacy of TLT in a much larger cohort was considered feasible.

\section{Treatment procedure}

In both of the NEST trials, all patients received standard medical management for acute ischemic stroke and all underwent the identical NeuroThera Laser System (NTS) procedure (PhotoThera, Inc., Carlsbad, CA, USA). Each NTS device contained a preprogrammed randomization code to determine whether the treatment was active or sham. Patients, technicians and clinicians were blinded to treatment allocation. The sham procedure was identical in every way to the active procedure except that no laser energy was delivered to the patient. The probe was mildly cooled during the application to ensure investigator and patient blinding to treatment group.

The NTS is an investigational device designed to provide noninvasive, transcranial laser treatment. The NTS is a portable device that uses class IV laser to deliver energy via a fiber optic cable to a handheld probe. Class IV lasers can burn the skin, in addition to potentially devastating and permanent eye damage as a result of direct or diffuse beam viewing. Hence, the patient and technician are both required to wear protective eyewear (due to the potential retinal damage if the laser is applied directly through the eye) and the treatment is administered in a room with restricted admittance during therapy. The laser probe was placed on the shaved head of the patient by a trained technician. The laser wavelength is in the near-infrared portion of the electromagnetic spectrum $(808 \mathrm{~nm})$ and invisible to the naked eye. The energy produced is non-ionizing and not associated with the risks of ionizing radiation. A complete treatment regimen consisted of applying the device to 20 pre-specified scalp locations, as identified by a cap placed on the patient's head, for two minutes at each site. The system was designed to deliver energy equally over the entire surface of the cerebral cortex regardless of stroke location. Preclinical experiments indicated that optimal amounts of energy penetrate the brain to a depth of at least $20-30 \mathrm{~mm}$, though energy may stretch across the brain to the opposite skull location based upon distribution of brain sulci and other tissue.

\section{Outcome measures}

The NIHSS was assessed at screening for entry into the study and again before randomization to treatment group. An examiner collected baseline factors including patient 
demographics, time to treatment, medical history and vital signs. After completion of the treatment procedure, patients entered a follow-up phase until either the patient decided to stop participation or the patient had participated in the study for 90 days. Outcome measures consisting of NIHSS and mRS were collected at the earlier part of day five or hospital discharge, and then again at 30,60 and 90 days.

\section{Statistical analysis}

All statistical analyses were performed post hoc using the Intention-To-Treat (ITT) population, with participant data analyzed according to assigned treatment at randomization. All hypotheses were tested at a 0.05 (2-sided) significance level with adjustments for multiple covariates.

The primary analysis was the mRS at 90-days with adjustment of covariates. For the primary analyses in NEST-1, in NEST-2, and in the present study of the pooled NEST-1 and NEST-2 data, the covariates were stroke severity (NIHSS score at baseline) and time from stroke onset (TFSO). These covariates were selected because of their prognostic association with stroke outcome. Baseline stroke severity was stratified into 3 subgroups (NIHSS score 7-10, 11-15, and 16-22), and time from stroke onset was stratified into two subgroups (0$12 \mathrm{~h}$. and 12-24 h.). The null hypothesis that the proportion of successes did not differ by treatment was tested using multiple logistic regression adjusted for stroke severity and TFSO.

In the pooled NEST-1 and NEST-2 data set, there were no baseline demographic or clinical variables that differed between the two treatment groups (the TLT-treated group and the sham group; Table 2), so no additional covariates were included in the primary analysis. A secondary analysis examined the difference due to treatment in the mRS distribution (on the 0 to 6 scale) using the Cochran-Mantel-Haenszel (CMH) test.

In the comparison of NEST-1 to NEST-2, baseline variables that differed significantly between the two studies were stroke severity (NIHSS score at baseline), TFSO, hypertension and ethnicity, while previous diabetes and previous stroke approached significance (Table 1). Although differences in ethnicity could be identified (many more 'other' in NEST-1), differences in the coding of ethnicity in the two studies made pooling the ethnic strata difficult. Thus, we included baseline NIHSS score, TFSO, hypertension, previous stroke and previous diabetes as covariates in a secondary analysis.

\section{Results}

As noted in Figure 1, a total of 780 patients were randomized into the NEST 1 and 2 trials. Two participants from NEST-2 population were excluded from the pooled analysis as one did not receive treatment, and the other withdrew consent before any follow up data was collected. Eight patients were lost of follow up and they had their last observation carried forward. Final pooled analysis was performed on a total of 778 patients (368 Sham, 410 TLT).

NEST-1 and NEST-2 trials were compared by treatment modality to assess for study differences between each study and within each treatment population. Table 1 notes the baseline characteristics of patients for each treatment modality comparing NEST-1 to NEST-2. Although baseline characteristics were generally well matched, there were some noted differences between the patient populations. For TLT therapy, there were differences in ethnicities, due to higher enrollment of 'other' races in NEST-1 (55.7\% in NEST-1 vs. only $9.4 \%$ in NEST-2). In NEST-2 there were more patients with hypertension and diabetes, while mean time to treatment was significantly shorter (16.93 hours in NEST-1 vs. 14.62 
hours in NEST-2). Also, baseline mean NIHSS was higher in NEST-2 (12.25 in NEST-1 vs. 13.13 in NEST-2). Comparing the sham groups in NEST-1 and NEST-2, and comparing overall patients in NEST-1 and NEST-2, they showed similar differences. The pooled NEST 1 and 2 data showed no significant differences in baseline characteristics between the two treatment groups (Table 2).

The primary analysis was logistic regression of the dichotomous 90-day mRS ( 0 $2=$ success), at 90-days adjusted for stroke severity (NIHSS score at baseline) and time from stroke onset (TFSO). This analysis showed that the success rate was significantly higher in the TLT group than in the sham treatment group; a total of 167 (40.73\%) of the TLT group patients, compared to 119 (32.34\%) of the Sham group patients, had a mRS 0-2 at 90 days $(\mathrm{p}=0.003$, OR $1.67,95 \%$ CI 1.19-2.35). A graph of the pooled 90-day mRS is shown in Figure 2.

The secondary logistic regression analysis of the binary success endpoint adjusted for baseline NIHSS, TFSO, previous stroke, previous diabetes and hypertension did not change the p-value for the treatment effect (0.003). Using the entire ordinal scale of mRS outcomes from 0 to 6, the $\mathrm{CMH}$ analysis showed a significant difference between TLT and sham with a shift towards better outcomes with TLT $(\mathrm{p}=0.005)$. (Table 3) Subgroup analysis (Figure 3) suggests that patients with a baseline NIHSS $<16$ received the most benefit. The occurrence of SAE's was not significantly different between treatment groups (35.4\% TLT vs. $41.3 \%$ Sham; $\mathrm{p}=0.09)$. Mortality was not significantly different between treatment groups $(15.9 \%$ TLT vs. $16.6 \%$ Sham; $\mathrm{p}=0.85$ ).

\section{Discussion}

This pooled analysis of the two NEST trials helps support the current evidence that TLT performed within $24 \mathrm{~h}$ of stroke onset may likely improve stroke outcome, and could be a beneficial option for even more acute stroke patients presenting within the first $24 \mathrm{~h}$ of symptom onset. The clinical safety profile was excellent with no differences for serious adverse events. Pooling of the studies demonstrated that there were no significant differences in 90-day mortality or serious adverse events.

It is important to analyze why the original two trials reported different results. NEST-1 randomized in a 2:1 ratio and reached its primary outcome measure for success after enrolling 120 patients. Conversely, NEST-2 did not reach statistical significance despite enrolling far more patients in a 1:1 ratio. In analyzing each of these studies for outcome differences, it is likely that NEST-2 had poorer efficacy due to including patients with more severe strokes as demonstrated by difference in baseline NIHSS, and including subjects with more co-morbidities (previous stroke, diabetes, and hypertension). Each of these factors has been documented as leading to poor outcomes after stroke. ${ }^{16}$ Regarding the increased severity of patients in NEST-2, the initial NIHSS was higher than in NEST-1 (baseline NIHSS $=13.2$ vs. 12.2 overall; $p=0.03$ ). The severity of the population is also reflected in the 90 day mRS of sham group. Nearly $50 \%$ of the patients in the NEST-2 sham group had a 90 day $\mathrm{mRS} \geq 4$ vs. only $36 \%$ in NEST- 1 sham group; furthermore, mortality was close to double in NEST-2 (9.75\% NEST-1 vs. 17.4\% NEST-2), reflecting the poor outcome due to the stroke severity or perhaps the amount of co-morbidities of the patients enrolled in NEST-2. Additionally, as reported by Zivin et al post-hoc subset analyses demonstrated that those with the most severe strokes (NIHSS 16-22) seemed to benefit less than those with moderate or moderate-to-severe strokes. ${ }^{15}$ In addition to greater stroke severity, the NEST-2 population also had a greater incidence of previous stroke, diabetes, and hypertension. 
Interestingly, patients treated after $12 \mathrm{~h}$ of stroke onset appeared to receive more benefit than patient treated before $12 \mathrm{~h}$ but the difference was not statistically significant. This finding seems discordant with the general belief that neuroprotective therapies should be initiated as fast as possible if they were to be effective. This finding of a better efficacy with a delayed treatment might be attributed to the higher proportion of patients treated within $12 \mathrm{~h}$ in NEST trials that failed to detect a treatment efficacy. However, animal research suggests laser therapy as more 'restorative' than 'neuroprotective' therapy, with an underlying mechanism possibly being induction of neurogenesis. ${ }^{9}$ Still, the best therapeutic window for TLT remains unknown.

The biggest limitation of this paper is that it is not a single cohort of patients, randomized to ensure homogeneity across groups. Neither the pooled nor the subgroup analyses were prespecified and are therefore subject to post-hoc bias and confounding factors. Because of these factors, a larger confirmatory study is still required to prove efficacy or make further claims. A phase III randomized placebo control study, NEST-3, has begun. Beyond NEST-3, it will be necessary to determine if TLT can then be combined with other therapies. The combination of TLT and t-PA has shown to be safe and does not increase intracranial hemorrhage in an animal model. ${ }^{17}$ Because TLT seems to have a unique mechanism, it is hoped that combining thrombolytic and laser therapies could have synergistic benefits for future stroke patients.

It is not surprising that the pooled analysis of these two studies resulted in a statistically positive benefit for patients since NEST-1 was strongly positive and NEST-2 displayed a possible signal towards efficacy $(\mathrm{p}=0.09)$. With this pooled analysis, there is compelling evidence that TLT is safe, and strong additive evidence that TLT may be effective for the treatment of acute ischemic stroke. If the confirmatory NEST-3 trial proves the benefit of TLT, far more stroke patients will receive treatment and a radical change in the paradigm for acute ischemic stroke will be established.

\title{
Acknowledgments
}

\author{
Source of funding \\ Partial funding through NIH grant (3P50 NSO44148-07S2) \\ NEST 1 and 2 was supported by PhotoThera, Inc, Carlsbad, California.
}

\section{References}

1. Tissue plasminogen activator for acute ischemic stroke. The national institute of neurological disorders and stroke rt-pa stroke study group. N Engl J Med. 1995; 333:1581-1587. [PubMed: 7477192]

2. Hacke W, Kaste M, Bluhmki E, et al. Thrombolysis with alteplase 3 to 4.5 hours after acute ischemic stroke. N Engl J Med. 2008; 359:1317-1329. [PubMed: 18815396]

3. Lloyd-Jones D, Adams RJ, Brown TM, et al. Heart disease and stroke statistics--2010 update: A report from the american heart association. Circulation. 121:e46-e215. [PubMed: 20019324]

4. Kleindorfer D, Lindsell CJ, Brass L, Koroshetz W, Broderick JP. National us estimates of recombinant tissue plasminogen activator use: Icd-9 codes substantially underestimate. Stroke. 2008; 39:924-928. [PubMed: 18239184]

5. Ginsberg MD. Current status of neuroprotection for cerebral ischemia: Synoptic overview. Stroke. 2009; 40:S111-S114. [PubMed: 19064810]

6. Stemer AB, Huisa BN, Zivin JA. The evolution of transcranial laser therapy for acute ischemic stroke, including a pooled analysis of nest-1 and nest-2. Curr Cardiol Rep. 12:29-33. [PubMed: 20425181] 
7. Streeter J, De Taboada L, Oron U. Mechanisms of action of light therapy for stroke and acute myocardial infarction. Mitochondrion. 2004; 4:569-576. [PubMed: 16120415]

8. Leung MC, Lo SC, Siu FK, So KF. Treatment of experimentally induced transient cerebral ischemia with low energy laser inhibits nitric oxide synthase activity and up-regulates the expression of transforming growth factor-beta 1. Lasers Surg Med. 2002; 31:283-288. [PubMed: 12355575]

9. Oron A, Oron U, Chen J, et al. Low-level laser therapy applied transcranially to rats after induction of stroke significantly reduces long-term neurological deficits. Stroke. 2006; 37:2620-2624. [PubMed: 16946145]

10. Rochkind S, El-Ani D, Nevo Z, Shahar A. Increase of neuronal sprouting and migration using 780 nm laser phototherapy as procedure for cell therapy. Lasers Surg Med. 2009; 41:277-281. [PubMed: 19347939]

11. Detaboada L, Ilic S, Leichliter-Martha S, Oron U, Oron A, Streeter J. Transcranial application of low-energy laser irradiation improves neurological deficits in rats following acute stroke. Lasers Surg Med. 2006; 38:70-73. [PubMed: 16444697]

12. Lapchak PA, Wei J, Zivin JA. Transcranial infrared laser therapy improves clinical rating scores after embolic strokes in rabbits. Stroke. 2004; 35:1985-1988. [PubMed: 15155955]

13. Lapchak PA, Maher P, Schubert D, Zivin JA. Baicalein, an antioxidant 12/15-lipoxygenase inhibitor improves clinical rating scores following multiple infarct embolic strokes. Neuroscience. 2007; 150:585-591. [PubMed: 17942241]

14. Lampl Y, Zivin JA, Fisher M, et al. Infrared laser therapy for ischemic stroke: A new treatment strategy: Results of the neurothera effectiveness and safety trial-1 (nest-1). Stroke. 2007; 38:18431849. [PubMed: 17463313]

15. Zivin JA, Albers GW, Bornstein N, et al. Effectiveness and safety of transcranial laser therapy for acute ischemic stroke. Stroke. 2009; 40:1359-1364. [PubMed: 19233936]

16. Kent DM, Selker HP, Ruthazer R, Bluhmki E, Hacke W. The stroke-thrombolytic predictive instrument: A predictive instrument for intravenous thrombolysis in acute ischemic stroke. Stroke. 2006; 37:2957-2962. [PubMed: 17068305]

17. Lapchak PA, Han MK, Salgado KF, Streeter J, Zivin JA. Safety profile of transcranial nearinfrared laser therapy administered in combination with thrombolytic therapy to embolized rabbits. Stroke. 2008; 39:3073-3078. [PubMed: 18687999] 


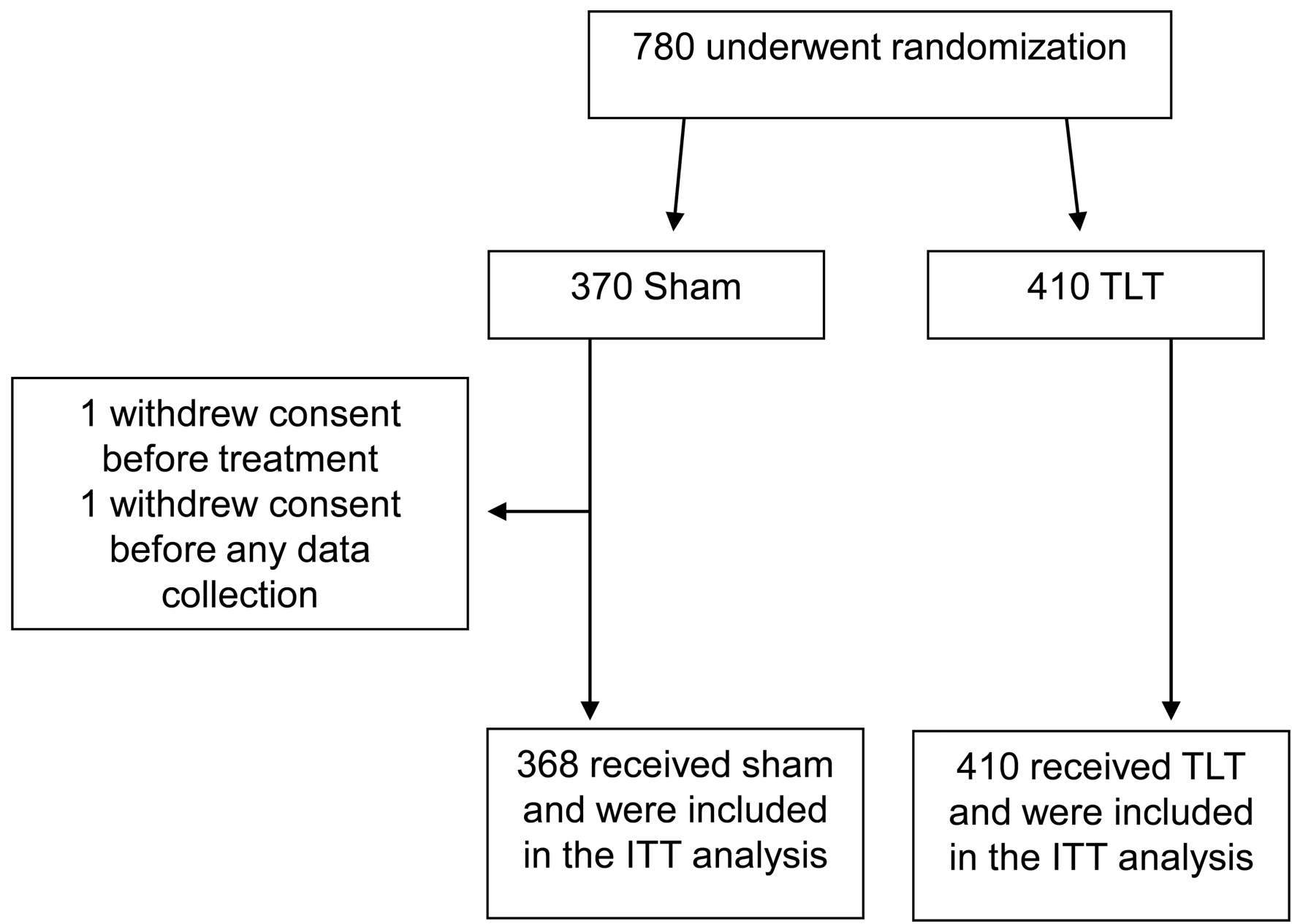

Figure 1.

Allocation of patients for this study (combined NEST 1 and 2) 


\section{Modified Rankin Scale}

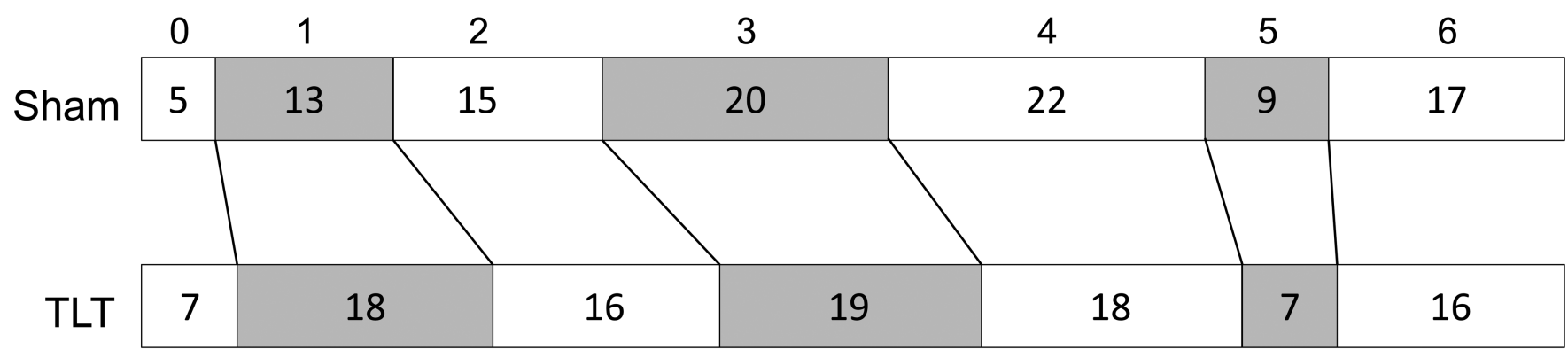

\section{Percentage of Patients}

Figure 2.

Primary outcome at 90 days according to scores on the mRS, in the intention to treat analysis. TLT was significantly better than sham by Cochran Maentel Haenszel test $\mathrm{p}=0.005$ 


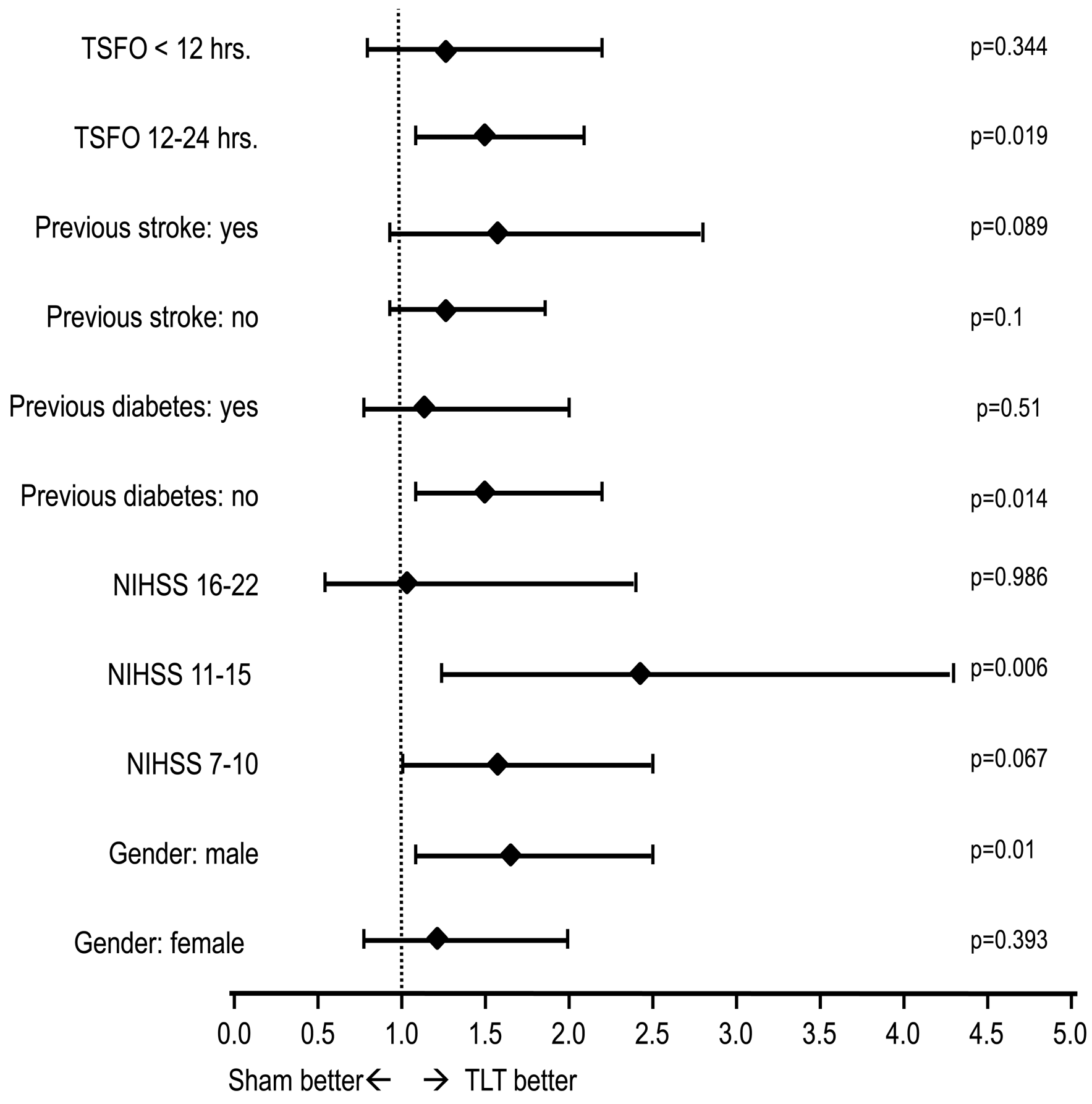

Figure 3.

Forest plot with unadjusted odds ratios for TLT as compared to sham for the 90 day mRS score of 0 to 2 on subsets of the data defined by categories selected from the baseline characteristics (Time from stroke onset (TFSO) to treatment, history of previous stroke, initial NIHSS and gender). The horizontal lines indicate 95\% CIs. P values for each stratum on the right. 


\begin{tabular}{|c|c|c|c|c|c|c|c|c|c|c|c|c|c|c|c|c|}
\hline \multirow{4}{*}{$\frac{\overline{0}}{\frac{\sigma}{0}}$} & \multirow{3}{*}{$\bar{z}$} & 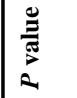 & $\begin{array}{l}8 \\
8 \\
0 \\
0 \\
0\end{array}$ & $\tilde{\delta}$ & $\begin{array}{l}\bar{\delta} \\
\dot{0}\end{array}$ & & & & & 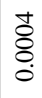 & $\begin{array}{l}\bar{\Xi} \\
0 \\
0\end{array}$ & $\frac{8}{\circ}$ & 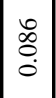 & $\begin{array}{l}\overline{8} \\
\dot{\nabla} \\
\dot{v}\end{array}$ & $\stackrel{8}{\circ}$ & \\
\hline & & 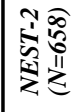 & $\frac{m}{2}$ & 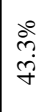 & & $\stackrel{\circ}{\stackrel{2}{ }}$ & $\stackrel{\circ}{\stackrel{\circ}{*}}$ & $\stackrel{\circ}{i}$ & $\frac{5^{\circ}}{6}$ & $\begin{array}{l}\stackrel{\bullet}{0} \\
\dot{ \pm}\end{array}$ & $\mid \begin{array}{c}2 \\
\hat{n} \\
m \\
\infty\end{array}$ & $\frac{\stackrel{\circ}{m}}{m}$ & $\left|\begin{array}{l}0 \\
\stackrel{0}{j} \\
\tilde{n}\end{array}\right|$ & $\begin{array}{l}\stackrel{0}{\circ} \\
\text { à } \\
\infty\end{array}$ & $\stackrel{n}{m}$ & \\
\hline & & 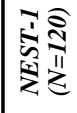 & $\begin{array}{l}n \\
0 \\
8 \\
8\end{array}$ & 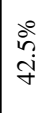 & & 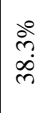 & $\stackrel{\text { ¿̊ }}{\mathrm{i}}$ & $\begin{array}{l}\circ \\
\text { in }\end{array}$ & 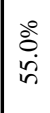 & $\stackrel{\overbrace{}}{\hat{\sigma}}$ & $\left|\begin{array}{l}0 \\
i \\
\infty \\
\infty\end{array}\right|$ & $\begin{array}{l}\text { ปे } \\
\text { ป̀ }\end{array}$ & $\frac{\stackrel{\circ}{\dot{d}}}{\stackrel{+}{d}}$ & $\begin{array}{l}\stackrel{0}{m} \\
\text { in }\end{array}$ & $\stackrel{\infty}{\stackrel{I}{I}}$ & \\
\hline & \multirow{3}{*}{ 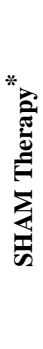 } & $\begin{array}{l}\frac{\mathscr{E}}{\pi} \\
2 \\
2\end{array}$ & \begin{tabular}{l}
$\infty$ \\
\multirow{0}{0}{} \\
0
\end{tabular} & $\vec{\sigma}$ & $\begin{array}{l}\bar{\delta} \\
\dot{\theta}\end{array}$ & & & & & 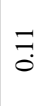 & ণे & $\stackrel{\overbrace{}}{0}$ & $\frac{\circ}{0}$ & $\begin{array}{l}\overline{8} \\
\dot{0} \\
\dot{v}\end{array}$ & $\frac{7}{0}$ & \\
\hline \multirow{6}{*}{ 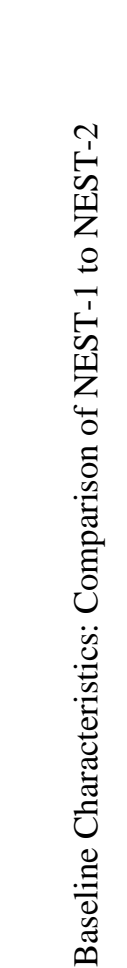 } & & 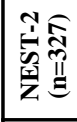 & $\therefore$ & $\begin{array}{l}\stackrel{\circ}{\circ} \\
\stackrel{\vec{\sigma}}{\vec{y}}\end{array}$ & & 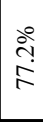 & $\stackrel{8}{\circ}$ & $\begin{array}{l}\text { do } \\
\text { ì }\end{array}$ & \begin{tabular}{|l}
$\infty$ \\
$\infty$ \\
$\infty$ \\
$\infty$
\end{tabular} & $\begin{array}{l}\stackrel{\theta}{b} \\
\dot{I}\end{array}$ & \begin{tabular}{|l}
$\stackrel{0}{0}$ \\
0 \\
$\omega$ \\
$\infty$
\end{tabular} & 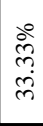 & 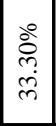 & $\begin{array}{l}\stackrel{0}{0} \\
\infty \\
\infty \\
\infty\end{array}$ & $\stackrel{=}{9}$ & \\
\hline & & 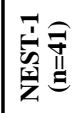 & $\mid \begin{array}{l}n \\
0 \\
\infty \\
0\end{array}$ & 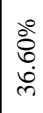 & & $\frac{\stackrel{0}{n}}{\dot{y}}$ & : & $\stackrel{\circ}{\circ}$ & $\begin{array}{l}\stackrel{\circ}{\circ} \\
\text { in }\end{array}$ & 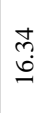 & $\mid \begin{array}{l}\stackrel{0}{\lambda} \\
\hat{\lambda} \\
\end{array}$ & $\begin{array}{l}\stackrel{2}{2} \\
\text { ते } \\
\text { ते }\end{array}$ & $\mid \begin{array}{l}\stackrel{0}{\alpha} \\
\alpha \\
\dot{v}\end{array}$ & $\begin{array}{l}\stackrel{\circ}{\circ} \\
\infty \\
o \\
\dot{\sigma}\end{array}$ & $\begin{array}{l}\stackrel{\mathrm{J}}{\mathrm{I}} \\
\mathrm{J}\end{array}$ & \\
\hline & \multirow{3}{*}{ 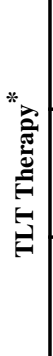 } & 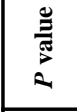 & $\delta$ & 8 & $\begin{array}{l}\bar{\delta} \\
\dot{0}\end{array}$ & & & & & $\bar{\delta}$ & $\mid \begin{array}{l}\vec{\Xi} \\
\dot{\vec{\theta}} \\
\dot{v}\end{array}$ & $\stackrel{m}{0}$ & 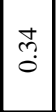 & $\begin{array}{l}\bar{\delta} \\
\dot{0} \\
\dot{v}\end{array}$ & $\stackrel{9}{0}$ & \\
\hline & & 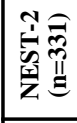 & 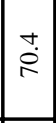 & $\begin{array}{l}\stackrel{8}{\circ} \\
\stackrel{+}{+}\end{array}$ & & $\stackrel{\circ}{\stackrel{2}{2}}$ & $\stackrel{\stackrel{\circ}{\perp}}{\stackrel{0}{=}}$ & $\frac{\stackrel{0}{c}}{\mathrm{c}}$ & | & $\begin{array}{l}\mathbb{S} \\
\dot{I}\end{array}$ & 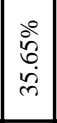 & 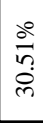 & $\mid \begin{array}{c}\stackrel{v}{q} \\
\stackrel{\sim}{\dot{m}}\end{array}$ & 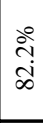 & $\stackrel{m}{m}$ & \\
\hline & & 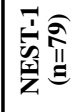 & 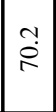 & 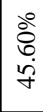 & & $\frac{\stackrel{\circ}{\hat{\delta}}}{\stackrel{8}{\circ}}$ & în & $\frac{\circ}{i n}$ & $\begin{array}{l}\stackrel{2}{2} \\
i n \\
i n\end{array}$ & 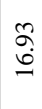 & $\begin{array}{l}0 \\
0 \\
0 \\
\text { di } \\
-1\end{array}$ & 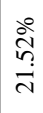 & 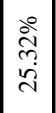 & $\begin{array}{l}\stackrel{0}{0} \\
\therefore \\
\text { in }\end{array}$ & 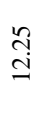 & \\
\hline & & 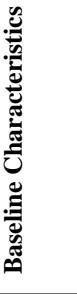 & $\begin{array}{l}\widehat{S} \\
\stackrel{0}{0} \\
\mathbb{4}\end{array}$ & 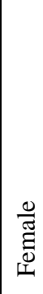 & 胥 & 愛 & 总 & 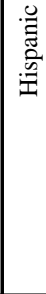 & 离 & 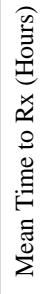 & 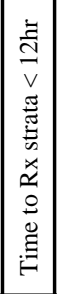 & 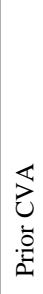 & 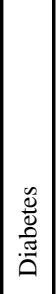 & $\begin{array}{l}\mid \\
Z \\
Z \\
Z\end{array}$ & 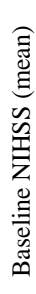 & \\
\hline
\end{tabular}


Table 2

Baseline characteristics: pooled data NEST 1 and NEST 2 by treatment modality

\begin{tabular}{|c|c|c|}
\hline & \multicolumn{2}{|c|}{ Pooled NEST $1 \& 2^{*}$} \\
\hline & $\operatorname{TLT}(n=410)$ & Sham $(n=368)$ \\
\hline Age $(y)$, mean, SD & $70.35 \pm 12.56$ & $69.72 \pm 11.85$ \\
\hline Female $(\%)$ & $184(44.88)$ & $152(41.3)$ \\
\hline Time to treatment, mean, SD & $15: 04 \pm 5: 45$ & $14: 52 \pm 6: 10$ \\
\hline Time to treatment strata $<12$ hours & $128(31.22)$ & $129(35.05)$ \\
\hline Baseline NIHSS score, mean, SD & $12.96 \pm 4.58$ & $13.04 \pm 4.5$ \\
\hline \multicolumn{3}{|l|}{ Baseline NIHSS score strata, No (\%) } \\
\hline Median & 12 & 12 \\
\hline 7 to 10 & $158(38.54)$ & $151(41.03)$ \\
\hline 11 to 15 & $122(29.76)$ & $97(26.36)$ \\
\hline 16 to 22 & $130(31.71)$ & $120(32.61)$ \\
\hline Previous stroke, no (\%) & $118(30.24)$ & $121(32.07)$ \\
\hline Diabetes, no (\%) & $124(30.24)$ & $118(32.07)$ \\
\hline Hypertension, no (\%) & $316(77.07)$ & $294(79.89)$ \\
\hline
\end{tabular}

* No statistically significant differences were noted in any characteristics between pooled treatment groups by t-test and Fisher exact test as appropriate.

Values are means $\pm \mathrm{SD}$ and $\mathrm{N}$ with percentages in parenthesis. 


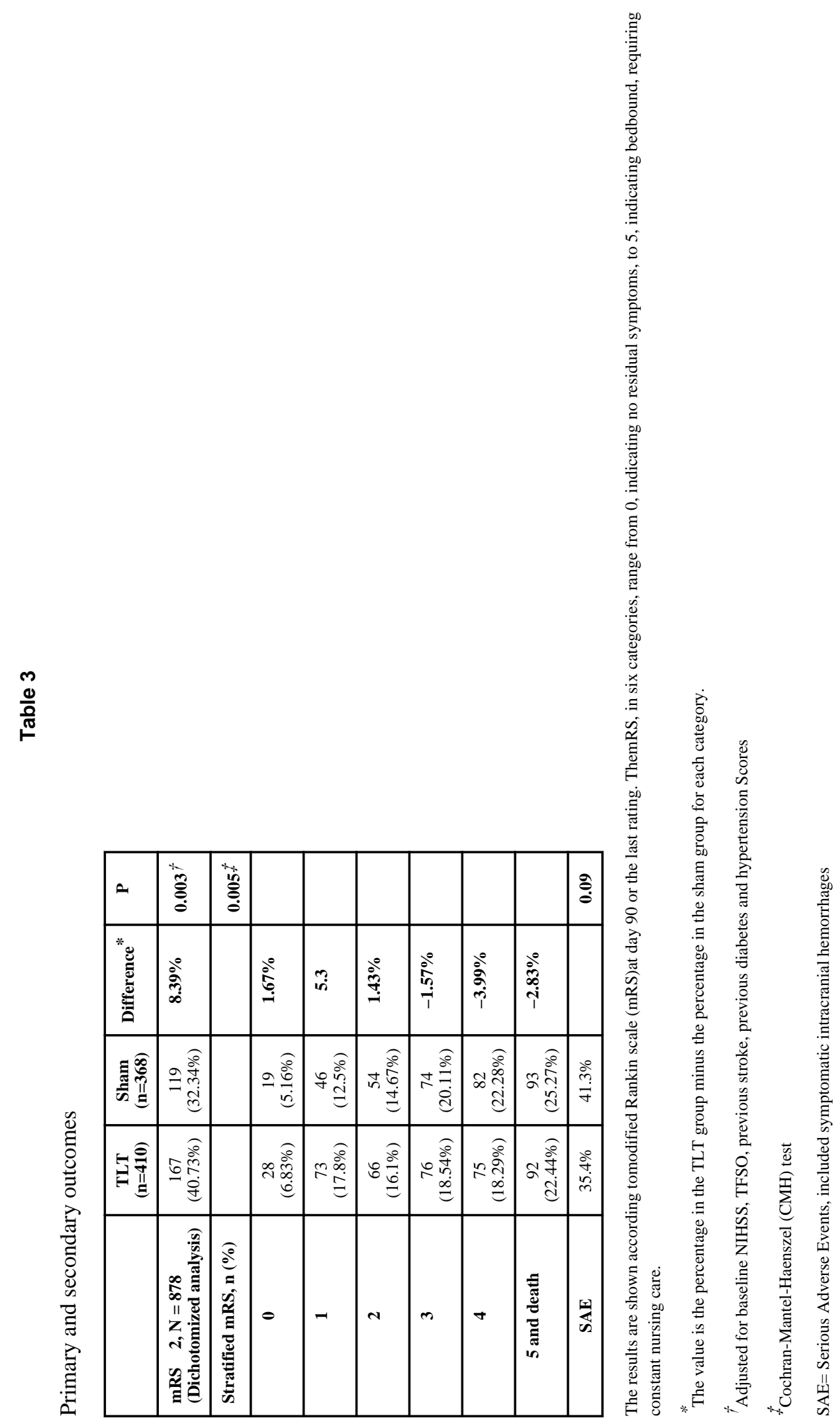

Int J Stroke. Author manuscript; available in PMC 2014 July 01. 